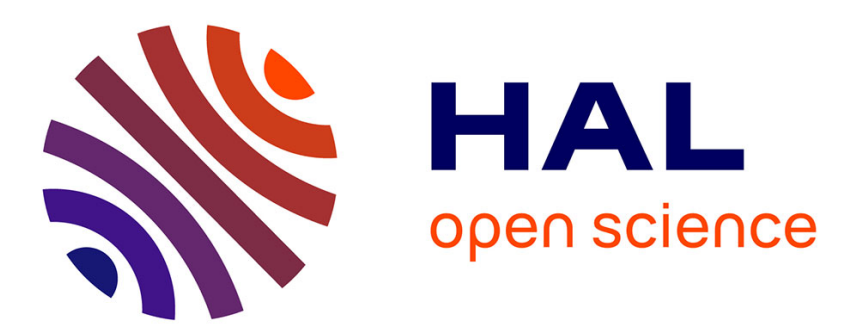

\title{
La mise en ouvre de la réglementation: une lecture économico-juridique du secteur électrique et des marchés publics
}

\author{
Thierry Kirat, Frédéric Marty
}

\section{- To cite this version:}

Thierry Kirat, Frédéric Marty. La mise en ouvre de la réglementation: une lecture économicojuridique du secteur électrique et des marchés publics. Economie et Prévision, 2005, accepté. A paraître. halshs-00004221

\section{HAL Id: halshs-00004221 https://shs.hal.science/halshs-00004221}

Submitted on 20 Jul 2005

HAL is a multi-disciplinary open access archive for the deposit and dissemination of scientific research documents, whether they are published or not. The documents may come from teaching and research institutions in France or abroad, or from public or private research centers.
L'archive ouverte pluridisciplinaire HAL, est destinée au dépôt et à la diffusion de documents scientifiques de niveau recherche, publiés ou non, émanant des établissements d'enseignement et de recherche français ou étrangers, des laboratoires publics ou privés. 


\section{La mise en œuvre de la réglementation : une lecture économico- juridique du secteur électrique et des marchés publics ${ }^{1}$}

Thierry Kirat

CNRS (IRIS-Université Paris-Dauphine)

thierry.kirat@dauphine.fr

Frédéric Marty

CNRS (GREDEG - Université de Nice Sophia-Antipolis)

frederic.marty@idefi.cnrs.fr

(11 avril 2005)

Dans le champ de l'analyse économique du droit et de la réglementation, la question de la mise en œuvre des règles est soit éludée, soit traitée sous l'angle de l'enforcement, entendu comme contrôle du respect de règles impératives (Polinski et Shavell, 2000 ; Shavell, 1993).

Si l'on considère les règles sous un angle plus ouvert, il est possible de rendre compte aussi bien des règles substantielles, qui définissent des droits et obligations, que des règles procédurales, qui organisent les conditions de leur mise en œuvre. Cet élargissement de perspective est d'autant plus justifié que le sens des règles - directive européenne, loi ou règlement - n’est pas tout entier contenu dans leur formulation initiale. Les analyses inspirées de la philosophie du pragmatisme en sciences sociales (Bazzoli, 1999 ; Bazzoli et Kirat, 2003) ont montré que la mise en œuvre des règles n'est pas réductible à leur application par des agents individuels rationnels. Elle implique des opérations de traduction et de production de significations par les destinataires des règles (acteurs économiques, tribunaux, agences de régulation, services de l'Etat)

Cette perspective conduit à s'intéresser à deux grandes questions qui structureront cet article. Il s'agit, d'abord, de celle de la portée économique des architectures institutionnelles de la réglementation. L'intervention des organes de réglementation a, en effet, des conséquences sur le type de règles mis en œuvre. Il s'agit, ensuite, de celle du processus de mise en œuvre stricto sensu des règles. Celles-ci, comme le mettent en évidence certains juristes (Jeammaud, 1993), ne font rien par elles-mêmes. Leur sens pratique résulte, en fait, de processus d'interprétation - notamment jurisprudentielle - et de leur articulation avec d'autres règles qui leur sont fonctionnellement liées.

La perspective suivie ici est plus complémentaire que substituable avec l'économie de la réglementation. Dans ses différentes composantes, celle-ci met l'accent sur des questions de

\footnotetext{
${ }^{1}$ Nous remercions vivement Laure Bazzoli, Véronique Dutraive, Jean-Michel Josselin, Sabine Montagne et les rapporteurs de la revue pour leurs commentaires sur les versions antérieures de cet article.
} 
justification de l'intervention de l'Etat ou sur les limites qui la caractérisent. La légitimité de l'intervention publique est fondée sur les spécificités de certains biens et services. Les effets pervers de la réglementation sont au cœur de la théorie de la capture du régulateur, ou de celle du Public Choice. Il en va de même pour la nouvelle économie publique dont l'objet porte sur les caractéristiques informationnelles et comportementales de la relation entre réglementeur et réglementé. Elle définit les termes de schémas régulatoires optimaux dans différentes hypothèses de distribution de l'information, d'observabilité ou de vérifiabilité des variables importantes dans les relations entre un organe public et une entité privée, notamment dans le cas des marchés publics et des contrats de délégation de service public (Laffont et Tirole, 1993, Mougeot et Naegelen, 1993).

Nous nous proposons d'appliquer une grille de lecture institutionnelle, sensible aux dimensions économiques du droit, aux dispositifs juridiques relatifs au secteur électrique et aux marchés publics, en particulier de la défense. Ces domaines ont en commun d'être fortement réglementés, de mettre en jeu la présence de l'Etat dans l'activité économique et, à ce titre, d'être liés à la conduite de l'action publique. Deux dimensions seront privilégiées : d'une part, celle de l'architecture institutionnelle des systèmes de réglementation et des conditions dans lesquelles ils opèrent ; d'autre part, celle de la mise en œuvre des règles, dont nous verrons qu'elle constitue un processus plus complexe qu'une simple application de règles prescrivant des comportements.

Une première section sera consacrée aux logiques d'action des institutions de réglementation. La seconde abordera la question de la mise en œuvre des règles, et insistera sur l'importance des recours aux tribunaux et des interdépendances entre règles. La conclusion reviendra sur les questions méthodologiques que l'utilisation de matériaux juridiques en économie permet de poser.

\section{LES POUVOIRS DE REGLEMENTATION ET LES LOGIQUES D'ACTION DES INSTITUTIONS DE REGLEMENTATION}

Les pouvoirs des institutions de réglementation et leurs logiques d'action peuvent être abordées dans les termes d'une analyse institutionnaliste du droit. Celle-ci met trois points en exergue (Mercuro, 2000). En premier lieu, les activités et les comportements économiques sont orientés par la structures des règles qui les régissent, d'un double point de vue : de celui de la définition des identités des acteurs, de celui des positions d'action et de ses facteurs limitatifs. En effet, le cadre juridique définit les capacités légales des acteurs à exercer des pouvoirs, à accéder à des revenus, à pratiquer un certain niveau de tarification et de marge, à évaluer le capital, etc. (Covaleski, 1995) En deuxième lieu, le système économique et juridique est conçu comme un système d'interdépendances mutuelles, dans lesquelles les conflits entre intérêts ou préférences sont régulés par des institutions juridiques, notamment par les tribunaux. Enfin, la dynamique économique et institutionnelle opère comme un processus de reconstruction continue des droits, donc des ensembles d'opportunités et de choix ouverts aux acteurs.

La prise en compte des conséquences économiques de l'architecture institutionnelle de la réglementation peut tirer profit des analyses proposées par John R. Commons, au début du siècle dernier. En effet, les études qu'il a consacré, essentiellement dans ses écrits « mineurs » (techniques) aux utilités publiques dans l'Etat du Wisconsin en 1907, permettent de comprendre que la question pertinente des réformes réglementaires n'est pas celle du "plus ou moins de règles », mais celle des pouvoirs, des compétences, et des logiques d'action des institutions de réglementation. A cet égard,Commons a examiné attentivement les différences de conséquences pratiques des moyens d'action dont des institutions alternatives disposent pour veiller à la mise en œuvre des règles, et des voies de recours ouvertes aux agents qui contestent les règles mises en place (Commons, 1910 et 1924). 
La méthode de Commons peut inspirer une étude des systèmes réglementaires contemporains (Mercuro, Medema, Samuels, 2000), en complémentarité avec les perspectives microéconomiques en économie du droit et de la réglementation (Perlzman, 1989, Posner, 1992, Stigler, 1992). En s'attachant non pas à la conception de règles optimales mais à leur mise en œuvre concrète, il est possible d'appliquer une telle analyse à la réglementation des industries de réseaux et aux marchés publics. .

\section{La libéralisation du secteur électrique comme déplacement des pouvoirs et des logiques}

Dans le secteur électrique, l'évolution récente du cadre réglementaire s'accompagne d'une redistribution des attributions des différentes institutions et de la création d'un régulateur sectoriel spécialisé.

Avant la libéralisation, tant le modèle américain du monopole privé local régulé que le modèle français du monopole public national verticalement intégré reposaient sur les prescriptions de l'économie du Bien-Etre. Dans le cas français, le cadre théorique de la gestion optimale des secteurs caractérisés par un monopole naturel, était fourni par les travaux de Maurice Allais (Allais, 1943). Ces derniers posaient les bases de la tarification au coût marginal et du modèle économique mis en place par Electricité de France (Boiteux, 1949 et 1956), fondé sur les théories de l'optimum de second rang.

Appuyée par la contestation des hypothèses à la base de la théorie du bien être (Laffont, 2000) et par les prescriptions issues de la théorie des marchés disputables (Baumol, Panzar, Willig, 1982), la libéralisation du secteur électrique, initiée par la directive européenne de 1996 et sa transposition nationale de 2000, visèrent à ouvrir le marché, en garantissant notamment l'accès du réseau aux tiers (Perrot, 1995). La question des modalités de construction de la nouvelle réglementation se pose alors. La construction de marchés concurrentiels peut-elle être le fruit d'une construction réglementaire ex ante (Deakin et Pratten, 1999) ou ne peut-elle résulter que d'une sédimentation jurisprudentielle, issue des décisions des tribunaux (Shavell, 1984)?

Le pool anglo-gallois, tel qu'il était organisé lors de la libéralisation du marché britannique de l'électricité en 1990, constituait l'exemple emblématique de la première approche. L'existence d'un pool de l'électricité centralisant les offres et les demandes et régulant le marché en surplomb (les négociations marchandes bilatérales restent marginales, à l'inverse du marché de l'électricité scandinave) reprend le modèle du commissaire priseur walrasien. Le modèle de régulation était en quelque sorte un modèle hybride fondé sur une concurrence totale sur certains niveaux de la filière électrique (production et distribution) et une régulation centralisée à l'échelon intermédiaire (transport). Le rôle de la réglementation est de s'assurer de la perfection de la structure de marché. Il s'agit de corriger les distorsions de concurrence et de s'assurer du respect de toutes les hypothèses sous jacentes au modèle. La réglementation doit alors œuvrer dans le sens de la création de marchés complets.

Cependant, la réglementation spécialisée, devrait, en toute logique, s'effacer dès lors que la construction du marché sera définitivement achevée et consolidée. Il s'agirait d'une régulation ex post principalement basée sur le droit de la concurrence, à l'instar de la Nouvelle-Zélande dont le régulateur des télécommunications est devenu une chambre spécialisée du conseil de la concurrence.

Alors que, du fait de l'hétérogénéité des situations de départ et des degrés de liberté accordés aux états membres en matière de transposition de la directive de 1996, les premières années de la libéralisation ne s'étaient pas traduites par une uniformisation des schémas institutionnels (Glachant, 2000), l'éventail des solutions régulatoires ne s'est pas avéré, au final, aussi large qu'il aurait pu l'être. Il tend même à se resserrer avec le temps. En outre, la Commission Européenne, instruite par les retours d'expériences, s'oriente peu à peu vers un modèle de réglementation unique reposant sur un accès réglementé des tiers au réseau, une dé-intégration verticale de l'opérateur historique (unbundling) 
séparant, au moins de façon comptable, les segments concurrentiels du réseau (lequel présente toujours des caractères de monopole naturel). Le développement de ce modèle de réglementation repose sur l'entrée en scène d'une nouvelle institution, le régulateur indépendant.

La création d'un régulateur sectoriel dans le domaine électrique, même si elle fait suite au précédent des télécommunications, doit être appréciée à sa juste valeur en terme d'innovation institutionnelle. Les autorités sectorielles de régulation induisent une rupture vis-à-vis de la régulation appliquée à EDF dans le cadre du précédent modèle de réglementation. Outre les questions relatives aux capacités de l'Etat actionnaire dans la gouvernance des entreprises, une confusion existait entre les différentes tutelles qui s'exerçaient sur EDF, notamment celles des finances et de l'industrie. L'entreprise publique se situait dans un modèle de régulation dans lequel un agent faisait face à deux principaux. Cette situation se traduit par un net affaiblissement de la tutelle (Laffont et Tirole, 1993). De plus, les trois objectifs de l'Etat (actionnaire, régulateur et redistributeur) rentraient en conflit. Le mélange des genres et des objectifs, entre l'entreprise nationale et la tutelle, alla jusqu'à la décision unilatérale d'EDF de pratiquer une péréquation tarifaire pour la Corse. Ce schéma classique de régulation administrative va être bouleversé par la mise en place d'autorités indépendantes de régulation (du Marais, 2003).

Il convient d'insister sur les pouvoirs quasi-juridictionnels et quasi-réglementaires des autorités de régulation. Ces derniers renforcent l'influence des décisions des “juges” dans la construction, la précision et la mise en œuvre de la réglementation. Les saisines des acteurs économiques en matière de réglementation doivent en effet être resituées par rapport aux possibilités d'action ouvertes par la création récente des autorités administratives indépendantes. Qu'elles soient à compétence transversale (Conseil de la concurrence) ou sectorielle (Autorité de Régulation des Télécommunications, Commission de Régulation de l'Energie,...), ces dernières fournissent aux parties prenantes des ressources juridiques leur permettant d'influer sur la réglementation. Elles jouent, en effet, un rôle déterminant dans le processus de mise en œuvre, de précision et d'approfondissement des règles. A la fois lieu de règlement de litiges privés de nature patrimoniale et de développement de politiques publiques visant à construire des marchés concurrentiels, ces autorités offrent la possibilité aux acteurs économiques de participer à la construction de la réglementation par la mobilisation des voies de saisines consultatives et contentieuses (Boy, 2000). Les parties prenantes du secteur régulé, au premier rang desquels les nouveaux entrants, disposent désormais de ressources juridiques leur permettant de peser sur la dynamique de la réglementation au travers de la jurisprudence. Par exemple, la possibilité ouverte à l'ART (par la loi de libéralisation du secteur des télécommunications du 26 juillet 1996) de trancher les litiges survenant entre les opérateurs crée au minimum des effets normatifs indirects dans la mesure où la solution dégagée pour régler un conflit a vocation à être appliquée aux cas comparables pouvant lui être soumis dans le futur (Marimbert, 2001). Il en va de même pour la Commission de Régulation de l'Energie, créée par la loi du 10 février 2000, dotée d'une fonction consultative sur la régulation du secteur, sur les tarifs d'utilisation des réseaux et sur l'évaluation des charges de service public. Elle est amenée, de par ses attributions, à trancher des litiges entre les gestionnaires de réseau et les utilisateurs. Elle est, de ce fait, habilitée à sanctionner les infractions à la régulation sectorielle. De telles compétences posent un problème de coordination avec le Conseil de la concurrence, lequel conserve son rôle de sanction des comportements anticoncurrentiels. La loi prévoit un mécanisme de saisines croisées permettant aux deux "juridictions" de travailler de concert. Les liens entre les deux autorités visent à garantir la complémentarité entre la régulation horizontale (i.e. sectorielle) et la régulation verticale (i.e. à compétence générale).

A l'instar de leurs homologues américaines, les autorités de régulation françaises cumulent des compétences exécutives et juridictionnelles et une indépendance vis-à-vis de l'Etat. Cependant, elles ne bénéficient pas du pouvoir réglementaire exclusif dont sont dotées les commissions outreAtlantique. Ainsi, une répartition originale des rôles entre tutelle et autorité de régulation se dessine en 
France. La régulation est considérée comme correspondant à la mise en œuvre concrète de la réglementation. Comme l'écrit l'ancien président de l'ART, Jean-Louis Hubert, " il ne faut pas confondre la réglementation et la régulation... La réglementation, c'est l'établissement du cadre juridique dans lequel doit s'exercer la régulation (...) La régulation, ce n'est pas la définition, c'est l'application du cadre général ; c'est le point de cohérence entre une réglementation stable et un marché en évolution. » (cité par Custos, 2002)

\section{La pluralité des règles et des logiques d'action dans les marchés publics}

La réglementation des marchés publics présente deux particularités par rapport à celles d'industries de réseau de service public.

En premier lieu, elle ne fait pas l'objet de l'action univoque d'un organe déterminé de contrôle ayant une compétence exclusive. Elle est plutôt exercée dans un cadre multiagents, au sein duquel chaque organe est doté d'une position d'action, définie par les règles dont il est tenu de faire observer le respect, et par la logique d'action qui découle de la mission qui lui est assignée. Le maillage des règles, des organes et des dispositifs tissé autour de la passation, de l'exécution et du règlement d'un marché public, interdit de le réduire une relation bilatérale nouée soit entre un agent réglementeur et un agent réglementé, soit entre un organe de contrôle et un organe de passation de marchés.

En deuxième lieu, les directives communautaires relatives aux marchés publics du début des années quatre-vingt-dix ${ }^{2}$ relatives à leur libéralisation ont certes introduit des possibilités (juridiques) de renforcement de la concurrence, mais elles n'ont pas radicalement affecté deux pivots du système réglementaire français : le droit des marchés publics et la comptabilité publique. En effet, un marché public demeure un contrat administratif, soumis à la comptabilité de l'Etat ou des collectivités territoriales. En droit interne, le renforcement de la concurrence par l'ordonnance du $1^{\text {er }}$ décembre 1986 qui soumet les personnes publiques au droit commun de la concurrence a certes renforcé les contraintes procédurales relatives à la publicité (publication des appels d'offre au JOCE) à la transparence de la passation des marchés, et aux responsabilités des entités adjudicatrices, mais les deux pivots du système réglementaires français n’ont pas été remis en question.

Dans ce système, remarquablement stable dans sa structure d'ensemble, le déroulement du processus de passation, d'exécution et de règlement du solde d'un marché public fait intervenir plusieurs catégories d'acteurs qui considèrent le contrat sous le prisme de leur position institutionnelle. En synthétisant les caractéristiques types à l'extrême, pour les marchés de l'Etat et des collectivités territoriales, le processus prend les modalités suivantes :

1. après définition des besoins et assurance de la disponibilité des ressources budgétaires, la personne publique lance une procédure de publication de l'appel d'offres, soit concurrentiel, soit négocié,

\footnotetext{
${ }^{2}$ Les nombreuses directives relatives aux marchés publics prises depuis 1990 ont été consolidées dans la directive 2004/18/CE du 31 mars 2004 relative à la coordination des procédures de passation des marchés publics de travaux, de fournitures et de services.
} 
2. l'examen des offres est assuré par une commission d'appel d'offre si la procédure est concurrentielle, par le service acheteur si la procédure est négociée (dans ce dernier cas, le processus se poursuit jusqu'à la réalisation d'un accord sur les termes du contrat),

3. jusqu'à l'entrée en vigueur du Code des marchés publics de 2004, tout projet de marché dépassant certains seuils était obligatoirement soumis à un examen préalable d'une commission spécialisée des marchés (CSM), et, pour les marchés des collectivités locales, au contrôle de légalité a posteriori par le préfet, le visa du contrôleur financier intervenait après que la CSM ait formulé son avis sur le marché,

4. après passation du marché, les services de l'Etat assurent le contrôle technique de l'exécution : soit par les services techniques de l'administration ou de la collectivité territoriale (les marchés de la Défense étant soumis à une réglementation spécifique),

5. la réception des travaux autorise le règlement du solde du marché, après service fait, par le comptable public.

Dans cet enchaînement-type, trois logiques de contrôle se succèdent : un contrôle juridique de la régularité de la procédure de passation, un contrôle technique de l'exécution, un contrôle financier. Or, chacun est exercé par un organe dédié, dont l'intervention n'est pas susceptible d'empiéter sur les prérogatives des autres. De plus, la mise en œuvre des dispositifs de contrôle ex ante au sein d'une même institution peut être différenciée : c'est le cas des commissions spécialisées des marchés, qui interviennent sur les marchés de l'Etat. En effet, nous avons montré que les logiques de contrôle $a$ priori mises en œuvre par les CSM sont largement structurées par le cadre de la passation des projets de marché examinés (Kirat, Marty, 2003). Dans le domaine où les marchés sont passés dans le cadre de procédures d'appel d'offres concurrentiels, comme c'est le cas pour les marchés publics de travaux qui relèvent de la CSM «Bâtiment et génie civil » (BGC), la logique d'évaluation de la commission est orientée vers le contrôle de la régularité formelle de la procédure de passation ; la théorie implicite de la CSM consiste à considérer qu'un marché qui est produit d'une mise en concurrence sera un marché efficace. A l'inverse, la logique d'évaluation mise en œuvre par la commission "Aéronautique, matériels électriques, mécanique et armement» (AMA) qui traite quasi exclusivement de marchés négociés de gré à gré, consiste en un examen attentif du montage contractuel proposé, en considérant des conditions d'exécution qu'il laisse présager. Dans ce cadre, le jugement de la CSM est davantage centré sur l'appréciation de l'efficacité attendue du montage contractuel dans l'exécution future.

Le contrôle de l'exécution du marché est assuré par les services de l'Etat, mais dans des dispositifs réglementaires particuliers selon le type de marché. Les marchés de travaux et les marchés industriels, notamment de la défense, ne sont pas équivalents de ce point de vue, dans la mesure où les services de l'Etat détiennent un pouvoir de direction ordinaire dans les premiers, et des pouvoirs de surveillance renforcés dans les seconds. En effet, une réglementation spéciale donne aux services de l'Etat un pouvoir d'accès aux documents comptables de l'entreprise dans les marchés de la défense, en vue de la réalisation d'éventuelles enquêtes de coût. Toutefois, cette réglementation est un genre de Janus : en même temps qu'elle donne à l'administration une prérogative d'accès aux données comptables des entreprises, elle neutralise les effets des enquêtes sur les conditions du règlement du marché par le comptable public (Kirat, Bayon et Blanc, 2003).

Enfin, le contrôle financier est exercé en considération, non pas de l'opportunité de la dépense publique ou de la réalité des coûts et des performances de l'entreprise, mais de la régularité de la procédure comptable, de la transmission par les services techniques au comptable public de pièces attestant de l'exécution du service, et de la disponibilité de crédits de paiement sur les lignes budgétaires d'imputation du marché.

Mis bout à bout et en relation sur la totalité des phases de la vie d'un marché public, l'ensemble des organes de contrôle et leurs logiques respectives d'intervention, dessinent un paysage inquiétant. 
L'absence d'un cadre de référence commun et de capacités d'audit économique, financier et technique intégré, conduit à l'existence d'un régime réglementaire des marchés publics ouvrant des opportunités (légales) de prélèvement de rentes sur l'Etat par ses fournisseurs. Dans le domaine des marchés de la Défense, le régime français de réglementation a été comparé avec des régimes étrangers, notamment celui des Etats-Unis. Dans ce dernier pays, les dispositifs en vigueur reflètent clairement la prégnance d'un principe d'accountability des services de l'Etat vis-à-vis du Congrès et, au-delà, du contribuable. Ce principe se traduit concrètement par l'existence d'une puissante agence fédérale d'audit des contrats de la Défense, d'une capacité d'expertise équivalente au sein de l'Office of Budget Management et du Government Accountability Office ${ }^{3}$, qui donnent à l'Etat fédéral des moyens de contrôle renforcés sur les fournisseurs de la défense (Kirat, Bayon et Blanc, 2003).

Par voie de conséquence, il convient de considérer la réglementation non seulement sous l'angle des énoncés qu'elle contient, mais aussi à l'épreuve de sa mise en œuvre.

\section{L'EXPERIENCE DE LA MISE EN GEUVRE ET L'EVALUATION DE LA REGLEMENTATION}

L'analyse de la réglementation passe aussi par l'évaluation des règles en situation. En effet, celle-ci suppose l'activation conjointe de règles et dispositifs pluriels, donc du «droit en action » et du travail d'interprétation. Les questions qui se posent alors sont celles de l'interprétation des règles par les acteurs publics (notamment les tribunaux administratifs et les régulateurs spécialisés) et par leurs destinataires privés.

\section{L'effectivité de la réglementation : de la définition de mécanismes régulatoires optimaux à l'épreuve des interprétations jurisprudentielles dans le secteur électrique}

Il convient de développer l'idée selon laquelle les origines de la réglementation ne se limitent pas à la définition ex ante d'un schéma régulatoire optimal, mais doivent être recherchées au travers des précisions et inflexions produites par la jurisprudence. Il en ressort qu'une analyse économique de la réglementation ne peut s'affranchir d'un travail sur un matériau de nature jurisprudentielle.

La montée en puissance d'une logique de régulation témoigne du fait que la dynamique des institutions, et plus particulièrement de la réglementation des services publics, ne procède pas seulement d'une construction législative et réglementaire, issue d'une théorie normative de l'intérêt général, mais au moins pour partie d'une construction par le jeu de la jurisprudence. Les actions et les stratégies des agents économiques par rapport aux règles (Weber, 1922) et l'activation des ressources que leur offre le droit, constituent l'un des principaux facteurs d'évolution de la réglementation.

En effet, les saisines des tribunaux en charge du contrôle de l'application de la réglementation par les agents économiques peuvent induire par la précision de certaines dispositions réglementaires encore floues ou par la constitution de cas de jurisprudence, une évolution progressive de la réglementation, pouvant constituer un approfondissement et / ou une inflexion de celle-ci. Une telle dynamique a été mise en évidence en matière de réglementation du secteur électrique (Marty, 2002).

\footnotetext{
${ }^{3}$ Nouvelle dénomination, depuis l'été 2004, du General Accounting Office.
} 
La dynamique de la réglementation des services publics a été appréhendée à partir de ses modalités de construction, de déploiement et d'application (Marty, 2002). La logique de la construction essentiellement jurisprudentielle du service public à la française, le long de la Troisième République, se retrouve dans le cadre de sa libéralisation. En effet, à partir des directives européennes et de leurs transpositions dans les lois nationales, les acteurs économiques se voient dotés de moyens d'actions pour saisir les tribunaux et faire évoluer par leurs décisions la nature de la réglementation.

L'influence de la jurisprudence sur la dynamique de la réglementation a été mise en évidence à travers une étude statistique réalisée sur une base constituée par le codage de 94 décisions, mesures conservatoires et avis du Conseil de la Concurrence dans le domaine des services publics entre 1988 et 2001. Elle permet de mieux saisir les liens entre la production jurisprudentielle de cette autorité administrative et la construction du nouveau cadre réglementaire ${ }^{4}$ (Marty, 2002). La méthode s'appuie sur le cadre général dressé par Korobkin (2001) en matière d'économie du droit empirique. Elle ne consiste pas dans l'analyse de la portée théorique d'un acte donné (ce qui est domaine de la doctrine juridique), mais en l'étude de l'ensemble des actes du Conseil relatifs aux services publics de façon à réaliser une exploitation statistique.

L'étude de la jurisprudence du Conseil de la concurrence permet de mettre en évidence un processus de construction jurisprudentielle de la réglementation qui met également en jeu les décisions de la Cour de Justice des Communautés Européennes. Par le jeu de sa jurisprudence issue des saisines contentieuses, le Conseil joue un rôle déterminant en matière de précision des modalités pratiques de la réglementation et de sa mise en œuvre. Qui plus est, son quasi-pouvoir réglementaire en fait un acteur central de la réglementation. Plus généralement, la place prise par les autorités administratives indépendantes procède des délégations accordées par le législateur, mais aussi et surtout des processus automatiques de régulation résultant de la production juridique de ces entités (Cohen, 1995). Cette production découle de leur activation par les acteurs économiques désireux de faire valoir leurs intérêts, voire de leur fonctionnement propre.

Il est possible en réalisant une analyse en composantes principales à partir des décisions et avis rendus par le Conseil de la concurrence, d'identifier les axes explicatifs fournis par les vecteurs propres (figure 1). L'axe 1 oppose les deux principaux griefs que le Conseil de la Concurrence peut notifier en application de l'ordonnance du 1er décembre 1986 (depuis codifiée dans le Code du commerce). Il permet donc de discriminer entre les abus de position dominante (article 8) et les cartels (article 7). L'axe 2 est constitué par l'opposition entre les dommages à une entreprise et les dommages à l'économie.

La projection à partir de ces deux vecteurs propres permet de dessiner quatre quadrants dans lesquels se répartissent les principales variables utilisées dans l'ACP.

\footnotetext{
${ }^{4}$ Deux voies de saisine sont ouvertes. La première, de nature consultative, correspond aux demandes d'avis. Elle est principalement utilisée sur le registre des "dommages à l'économie". La structure du marché ou le comportement de l'opérateur dominant permettent-ils l'existence d'une concurrence effective? La seconde voie de saisine, de nature contentieuse, correspond aux décisions et aux mesures conservatoires. L'action se situe alors sur le registre des "dommages à des tiers". Il s'agit de faire mettre un terme et de faire sanctionner les pratiques jugées anticoncurrentielles d'un des opérateurs du marché.
} 
Figure 1 - Essai d'identification d'une dynamique réglementaire à partir de l'activité du Conseil de la Concurrence

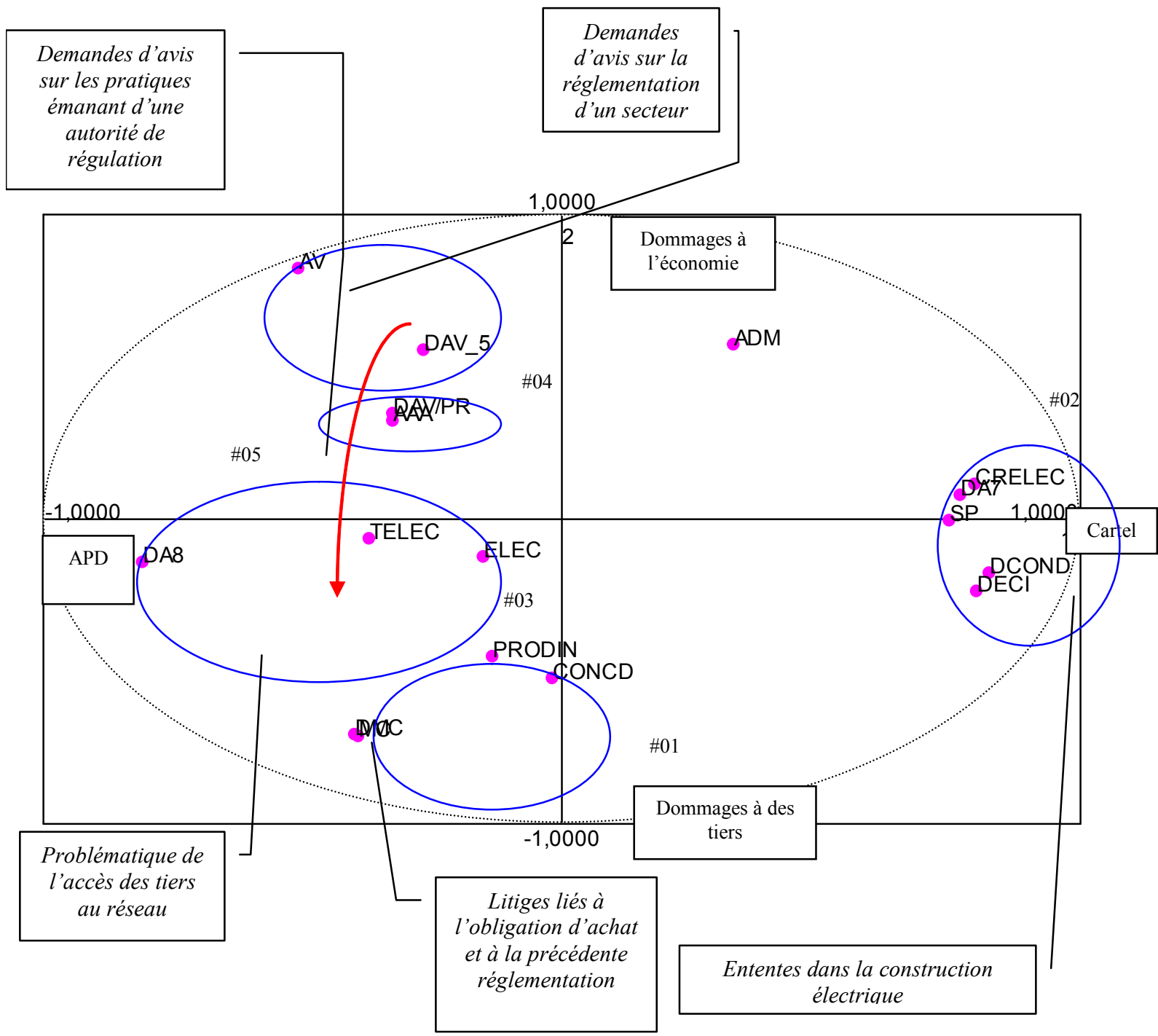

Identification des variables :

CRELEC : secteur de la construction électrique

TELEC : secteur des télécommunications

ELEC : secteur de l'électricité PRODIN : saisine émanant d'un producteur d'électricité non nationalisé

CONCD : saisine émanant d'un concurrent direct de l'opérateur historique

ADM : saisine émanant de l'administration

AAA : saisine émanant d'une autorité administrative indépendante

AV : avis du conseil de la concurrence

DMC : demande de mesures conservatoires

DECI : décision du conseil de la concurrence

SP : sanctions pécuniaires

DAV/ PR : demande d'avis sur les pratiques de l'opérateur historique

DAV_5 : demande d'avis sur la situation de la réglementation du secteur vis-à-vis des règles de concurrence

DA8 : demande d'avis au titre de l'article 8 de l'ordonnance du $1^{\text {er }}$ décembre 1986 (abus de position dominante), actuel article L

420-2 du Code du Commerce.

DA7 : demande d'avis au titre de l'article 7 (entente anticoncurrentielle), actuel article L 420-1 du Code du Commerce. 
Dans le quadrant bas gauche de la figure 1, nous retrouvons des demandes de mesures conservatoires visant à faire cesser des abus de positions dominantes commis par l'opérateur de réseau. En haut à gauche, il s'agit principalement de demandes d'avis quant à la situation générale de la concurrence dans un secteur ou quant aux pratiques de l'opérateur. Ces avis sont formulés par les autorités publiques en charge de l'intérêt général (administration ou autorité administrative indépendante). Dans le quadrant haut droit, sont présentes des saisines émanant des pouvoirs publics suite aux dommages causés à l'économie par des ententes de firmes de construction mécanique. En bas à droite, il s'agit de saisines de firmes s'estimant victimes d'une entente anticoncurrentielle.

La flèche de la figure 1 indique la dynamique réglementaire initiée par les télécommunications, qui peut évoquer un cheminement possible : chaque service public de réseau suivrait cette dynamique. A l'heure actuelle, à l'exception des télécommunications et de l'électricité, les services publics sont concernés par des avis du Conseil sollicités par l'administration quant à la situation actuelle de leur réglementation. Un pas supplémentaire est franchi (c'est le cas par exemple du gaz), lorsque le Conseil est sollicité sur la transposition d'une directive européenne. Ces avis prennent place dans une logique de basculement de la conception que l'on se fait de l'action publique et qui prend place dans une séquence dont on peut synthétiser les phases.

Dans un premier temps, les pouvoirs publics demandent au Conseil de la concurrence son avis sur la réglementation du secteur. Il s'agit d'estimer si les distorsions de concurrence liées à l'existence d'un monopole légal (abus de position dominante par excellence) ne causent pas un dommage à l'économie supérieur aux gains attendus de la nationalisation. Dans un second temps, le témoin passe aux autorités de régulation. Les demandes d'avis portent d'abord sur les modalités d'application des textes. Il s'agit de voir si les dispositifs techniques de réglementation du secteur sont conformes à la logique de la nouvelle réglementation. Dans ce cadre, la portée de la notion de dommage à l'économie est réduite dans la mesure où l'on ne considère plus que l'ensemble des firmes d'un secteur donné. Les avis de l'autorité de régulation s'attachent ensuite au comportement de l'opérateur dominant, aux entraves qu'il fait peser sur les nouveaux entrants pour l'accès à son réseau et aux avantages indus qu'il peut tirer de la maîtrise de l'infrastructure essentielle. Nous sommes ici très proches de la notion de régulation asymétrique. La réglementation est alors rabattue au contrôle de la stratégie de l'opérateur dominant. Nous sommes maintenant aux frontières du dommage à l'économie et du dommage à des tiers. L'autorité de régulation tend à défendre l'intérêt des concurrents de l'opérateur dominant. La réduction des rentes de ce dernier rapprochera le secteur de la situation de concurrence parfaite, considérée seule à même de permettre d'atteindre l'intérêt général. Enfin dans une dernière phase, l'application du nouveau cadre réglementaire se fait par des saisines contentieuses directes émanant des nouveaux entrants dans le secteur. Il s'agit alors de dénonciations d'abus de position dominante appuyées sur la notion de dommage à des tiers. Ainsi, l'activation des droits par les intérêts économiques privés permet d'achever la construction du nouveau cadre régulatoire.

Cette étude de la dynamique du changement réglementaire, tel qu'il peut être saisi au travers de l'action juridique, permet de mettre en lumière un phénomène de passage de relais dans le processus de basculement de la réglementation des services publics. Ce phénomène est porté par les saisines d'acteurs distincts, à partir d'un continuum allant de la notion de dommage à l'économie à celle de dommage à des tiers. L'analyse des recours des acteurs économiques devant le Conseil de la concurrence repose sur l'hypothèse que l'activation des droits donnés par la loi est à la fois un outil de connaissance (dans la mesure où l'action en justice induit une formulation des intérêts et des raisons d'agir) et un moteur de la dynamique du changement réglementaire. L'action des particuliers et des intérêts constitués devant les tribunaux et les autorités administratives indépendantes constitue donc bien à la fois un facteur d'application de la législation mais aussi de précision et d'évolution de celle-ci, via l'activation des textes généraux. 
Ce phénomène de construction de la réglementation applicable au secteur électrique au travers de sa mise en œuvre peut de la même façon être observé aux Etats-Unis. Si une telle logique d'évolution remédie aux inefficiences incitatives des schémas traditionnels de réglementation (Laffont et Tirole, 1993), elle n'en est pas moins potentiellement porteuse de nouveaux risques et coûts. Ces derniers tiennent au fait que ce mécanisme de tâtonnement réglementaire est d'autant plus marqué en matière de réglementation du secteur électrique que les facteurs d'incertitudes liés aux impacts des stratégies individuelles sur la réglementation se doublent d'une incertitude tenant à la dynamique réglementaire elle-même.

En effet, la réglementation du secteur électrique se présente à maints égards comme un cadre réglementaire encore "en chantier ». Cette caractéristique est à la fois liée au processus de libéralisation lui-même et aussi à la nature intrinsèquement « jurisprudentielle » de la construction de la réglementation. Il existe, en effet, une nouveauté radicale dans le projet de construire des marchés concurrentiels à partir de règles qui en définissent l'organisation. La définition de règles susceptible d'encadrer efficacement les marchés électriques est le fruit d'évaluations et d'inflexions ex post des impacts des règles et pas seulement la résultante de la définition ex ante d'un cadre réglementaire optimal. ${ }^{5}$

Cette logique est dans le même temps porteuse d'efficacité dans la mesure où elle permet de laisser ouvert l'éventail des possibles avant de déterminer, au regard des retours d'expériences, le schéma réglementaire le plus efficace. Dans cette perspective, il est intéressant de noter que le régulateur américain, la FERC, a longtemps laissé de larges degrés de liberté aux Etats pour définir leur modèle propre de régulation de l'industrie électrique. Si cette application de la subsidiarité a grandement nuit aux échanges d'énergie inter-Etats, elle n'en a pas moins permis de favoriser l'émergence de plusieurs schémas de régulation profondément distincts. Cette autonomie, ironiquement baptisée « des 100000 fleurs »(Cramton, 2003), a permis de dégager les schémas les plus efficaces, lesquels ont inspiré le modèle unique de régulation proposé par la FERC en 2002. Ces propositions tirent les principales leçons de la défaillance des marchés californiens et amendent le schéma réglementaire mis en place dans le Nord-Est avec le PJM (Pennsylvanie-New Jersey-Maryland). Il s'agit notamment d'accorder une place centrale sur les marchés aux contrats à terme, laissant au marché au comptant un rôle résiduel. Parallèlement les offres sur le marché spot se feraient la veille pour le lendemain, heure par heure et engageraient la responsabilité des offreurs, ce qui n'était pas le cas en Californie. La sécurité du système serait renforcée par la création d'un marché pour les congestions et par la rémunération des unités non appelées mais constitutives de la réserve de sécurité (Joskow et Tirole, 2004).

Les expériences américaine et européenne témoignent donc d'une telle logique de construction de la réglementation conçue comme un double processus d'essais et d'erreurs, de capitalisation des retours d'expérience (Borenstein, 2002) et d'arbitrage dans les conflits d'intérêts des diverses parties au système électrique. Il convient maintenant de déterminer si une telle évolution, reposant sur des mécanismes de tâtonnement réglementaire comparables, peut se retrouver en matière de

${ }^{5}$ Comme l'observe à juste titre J.-P. Fitoussi : « Cette indétermination des règles futures d'organisation des marchés est due à la nouveauté de la situation : la transition vers le marché est une chose inédite, ce qui oblige à ce que l'organisation mise en place doive être réformée, au fur et à mesure que son fonctionnement effectif révélera ses défaillances» (Fitoussi, 2003). 
réglementation des marchés publics. A cette fin, nous envisagerons la situation des marchés français de la défense.

\section{La mise en œuvre de la réglementation dans le contrôle des coûts des marchés publics de la défense}

Les marchés de la défense sont sujets à des dispositions particulières par rapport au droit commun des marchés publics, dans la mesure où la question du contrôle des prix de revient est a priori plus importante dans des domaines techniques complexes liés au régalien que dans les activités de production de biens ou de services plus routinières. L'un des principaux enjeux de ces marchés est de fixer une formule de prix capable d'inciter le fournisseur à l'efficacité et de répartir les risques entre l'Etat et l'entreprise. Les propriétés économiques des formes classiques de prix, (forfaitaires, à remboursement de coûts ou incitatifs) sont bien connues dans la littérature (Laffont et Tirole, 1993, McAfee et McMillan, 1986, Scherer, 1964). Il convient cependant de s'assurer que le fonctionnement concret des formules de prix correspond aux schémas théoriques, surtout au regard de la structure non concurrentielle des marchés d'armements. En effet, le contrôle des coûts effectifs est central dans les marchés de type Cost-Plus ${ }^{6}$, mais il l'est aussi dans les marchés à prix forfaitaire conclus dans un cadre non-concurrentiel: la vérification de la véracité des informations justifiant le niveau du prix proposé par la firme est à ce titre une nécessité pour l'acheteur public.

Le régime juridique du contrôle des coûts de revient des marchés publics de l'Etat est fixé par le Code des marchés publics, et précisé techniquement par des textes dénués de valeur réglementaire, c'est-àdire des instructions ou circulaires, remontants à plusieurs décennies. Nous avons pu mettre en évidence que les dispositifs en question sont empiriquement fragiles, et que leurs destinataires sont engagés dans une volonté de maintenir le régime du contrôle des coûts de revient dans cet état (Kirat, Bayon et Blanc, 2003).

Le Code des marchés publics du 7 mars 2001 prévoit, dans son article 126, un droit d'accès de l'autorité publique aux comptes des entreprises titulaires de certains marchés, notamment ceux qui sont marqués par l'importance du secret ${ }^{7}$; mais il ne s'agit là que d'une faculté ouverte aux services de l'Etat, qu'il faut mettre au regard de l'article 127 prévoyant que ce droit d'accès est conditionné à l'insertion dans les documents contractuels de la référence aux obligations prévues à l'article 126. En

${ }^{6}$ Il s'agit de marchés dans les lesquels le contractant se voit garantir d'une part le remboursement des coûts encourus pour réaliser la prestation et d'autre part une rémunération "raisonnable" des capitaux immobilisés. De cette structure binôme naît l'appellation Cost plus (fees). Pour une évaluation de ces contrats, le lecteur pourra se reporter à Laffont et Tirole (1993).

${ }^{7}$ Ces dispositions, qui concernent « les marchés de travaux, fournitures ou études pour lesquels la spécialité des techniques, le petit nombre d'entreprises compétentes, des motifs de secret ou des raisons d'urgence impérieuse ne permettent pas de faire appel à la concurrence ou de la faire jouer efficacement », sont identiques, à un alinéa près, à celles de l'article 233 de l'ancien Code. La différence entre l'art. 233 de l'ancien Code et l'art. 126 du Code de 2001 réside dans le fait que la disposition donnant au ministre des finances et au ministre chargé du secteur intéressé le pouvoir de définir par arrêté les documents comptables exigibles du cocontractant a disparu dans l'art. 126. 
d'autres termes, il n'y a de possibilité de contrôle qu'à la condition que celui-ci ait fait l'objet d'un accord entre les parties, c'est-à-dire qu'il soit autorisé par l'entreprise susceptible d'être contrôlée...

En dehors du Code, les textes de référence sont une instruction de 1964 et une directive de 1971.

L'instruction du 15 octobre 1964 prise au titre de l'application de l'article 54 de la loi nº3-156 du 23 février 1963 instituant un droit de contrôle des prix de revient pour certains marchés prévoit une possibilité de contrôle des marchés publics négociés de gré à gré ; toutefois, l’instruction limite la portée du contrôle avec ces deux dispositions :

- l'administration n'est pas tenue d'exercer un contrôle : elle n'use de ce droit de contrôle que si elle le juge opportun ;

- le droit de contrôle ne concerne que les seuls contrats conclus : " ce droit naît donc après la conclusion des marchés. Il en résulte tout d'abord qu'aucun contrôle ne peut être exercé avant la conclusion du marché (il ne peut donc servir pour la fixation initiale des prix de celui-ci); en second lieu, le contrôle ne peut être utilisé pour remettre en cause le contrat initial, et notamment les conditions de prix que celui-ci prévoit » (Instruction du 15 octobre 1964, J.O. du 30 octobre 1964, p. 134).

L'instruction fait du contrôle un instrument a posteriori, dont la portée mérite discussion. Ce texte présente la vocation du contrôle a posteriori comme permettant de calculer les prix définitifs dans les marchés à prix provisoires ou d'accumuler des informations qui pourront être utiles dans passation de marchés ultérieurs sur la même prestation ou sur une prestation dérivée de la première (comme la commande de pièces détachées d'un ensemble).

La portée de l'instruction du 15 octobre 1964 est limitée, dans la mesure où le contrôle des coûts de revient n'est pas pensé comme un instrument instituant une capacité des organes publics acheteurs de réduire leur asymétrie informationnelle vis-à-vis de leurs fournisseurs en matière de coûts de revient des prestations.

L'étroitesse du contrôle a posteriori organisée par l'instruction de 1964 est confirmée dans une circulaire du 26 juillet 1971 relative à la pratique des analyses de coûts dans les marchés publics: cette circulaire précise que le contrôle peut être réalisé si l'administration le juge opportun, mais qu'il ne doit pas être de nature à remettre en cause le marché existant. Il ne peut servir qu'à dégager des «éléments d'information utiles à la négociation de contrats ultérieurs » (circulaire du 26 juillet 1971, p. 145). Quant à l'analyse a priori que cette circulaire évoque, ayant un caractère pré contractuel, elle ne peut être source d'obligations contractuelles du fournisseur de l'Etat en matière d'engagements à divulguer des informations comptables ou économiques. La circulaire précise laconiquement, sans prévoir de dispositifs concrets permettant de donner une force opératoire à ce principe, que l'analyse de coûts $a$ priori est « la condition nécessaire d'une négociation sérieuse des prix de contrats portant sur des prestations élaborées et [que] les acheteurs du secteur public doivent s'efforcer de la mettre en cuvre de façon plus systématique.» (Ibid., p. 145).

On peut, en définitive, constater que la mise en œuvre des dispositions du Code des marchés publics relatives au contrôle des coûts de revient s'avère moins contraignante que ce que pouvait laisser anticiper leur formulation. Il faut néanmoins préciser que l'Etat traitait il y quelques décennies avec des entreprises sous contrôle public. L'exercice des pouvoirs de la tutelle permettait d'accéder aux données de coût des établissements relevant d'une logique d'arsenal; mais dans un contexte marqué par le relâchement du lien tutélaire avec les fournisseurs d'équipements de défense, l'application des textes devient problématique : des textes conçus dans un système d'économie administrée ne sont plus réellement adaptés à un système libéralisé et n'atteignent pas le niveau requis par l'exercice d'un contrôle renforcé sur des monopoles privés. A cet égard, la profondeur des dispositifs du Code des marchés publics américain (Federation Acquisition Regulation), qui portent véritablement sur des 
monopoles privés réglementés, est loin d'être atteinte en France. Or, les perspectives d'évolution des dispositifs français relatifs au contrôle des coûts sont contraintes par les positions du Conseil d'Etat sollicitées par les parties intéressées dans l'industrie - sur des tentatives de changement de règles de la part des pouvoirs publics.

A titre d'exemple significatif, le ministère de la défense, ainsi que les ministères de l'économie et des finances, de l'industrie, des télécommunications ont pris en 1996 des arrêtés touchant aux conditions du contrôle des données relatives aux coûts de revient des firmes et à la définition d'une norme de forfaitisation des frais d'approvisionnement. Ces arrêtés durcissaient les termes du contrôle exercé par les autorités publiques au titre de l'article 223 de l'ancien Code des marchés publics ${ }^{8}$. L'objet des arrêtés était en la matière de poser des principes clairs quant aux obligations des entreprises en termes de transmission de données certifiées et de définir des règles de forfaitisation des dépenses d'approvisionnement relatives aux opérations générales d'achat et des frais hors production. A cet égard, l'article 5 de l'arrêté du 5 mars 1996 définissant le cadre général dans lequel sont déterminés les coûts de revient des prestations des sociétés intervenant dans le domaine aéronautique et spatial précisait que :

«... le contrôle des frais de production est effectué en fin d'exécution de chaque prestation ou groupe de prestations des marchés ou d'un programme visé à l'article 2, en utilisant directement le système de comptabilité générale et de comptabilité analytique d'exploitation de l'entreprise, ainsi que ses méthodes d'enregistrement des coûts et de suivi des affaires, tel que celui-ci est pratiqué à l'intérieur de l'entreprise par les responsables de ces affaires...

L'organisme fournit à l'administration... le descriptif, suffisamment détaillé... de son système comptable et de ses méthodes d'enregistrement des coûts et de suivi interne des affaires mentionnées à l'alinéa précédent ; ce descriptif précise notamment la délimitation de ses frais de production et de ses frais hors production....

Une contre-expertise sur le dispositif comptable et les méthodes d'enregistrement des coûts et de suivi interne des affaires peut être effectué à la demande et à la charge de l'administration ou du service enquêteur. » (art. 5 de l'arrêté du 5 mars 1996).

L'article 6 de l'arrêté prévoyait que «Les dépenses d'approvisionnement relatives aux opérations générales d'achat sont rémunérées forfaitairement »; l'article 7 disposait que le taux des frais hors production (comprenant notamment les frais de distribution, les frais d'administration générale, les charges financières et les dépenses d'études libres) «....est fixé forfaitairement ... en fonction des derniers frais hors production contrôlés pour chaque entreprise, ou par branche d'activité au sein de l'entreprise ».

Les entreprises d'armement, par l'entremise du Conseil des industries de défense française (CIDEF), ont obtenu l'annulation par le Conseil d'Etat de ces arrêtés. Les arrêts du Conseil d'Etat CIDEF N¹91514 et 191515 rendus en date du 17 décembre 1999 limitent considérablement le pouvoir de réglementation des marchés publics de la défense. Ces deux arrêts, en l'occurrence, retiennent l'argument avancé par le CIDEF selon lequel les ministres concernés, dont le ministre de la Défense, «

\footnotetext{
${ }^{8}$ Correspondant à l'art. 126 du Code de 2001.
} 
ont excédé le champ de l'habilitation qu'ils détenaient en vertu des dispositions ... de l'article 223 du Code des marchés publics notamment en édictant le principe d'une rémunération forfaitaire pour certains coûts de production et en en excluant d'autres du calcul des prix des produits vendus » (CE, CIDEF c/ ministre de la Défense, 17 décembre 1999).

L'annulation des arrêtés concernés par le Conseil d'Etat est significative de l'importance des contraintes, voire des impossibilités, pour le ministère de la Défense d'exercer un véritable contrôle économique des entreprises du secteur de l'armement et de définir des normes contractuelles équivalentes à celles pratiquées au Royaume-Uni et aux Etats-Unis. Tout se passe comme si la trajectoire institutionnelle du modèle français d'acquisition, largement régi par les règles des marchés publics, bloquait son évolution sur une dépendance du sentier qui confine le contrôle des contrats et des fournisseurs dans des marges d'action limitées.

\section{La régulation des dépassements de coûts dans l'exécution des contrats administratifs}

La question qui se pose maintenant est de savoir si les marchés de la défense constituent un cas spécifique, ou s'ils posent dans des termes particuliers les mêmes questions que les marchés publics «banals » en matière de régulation des dépassements de coûts.

La théorie microéconomique des marchés publics pose comme objet central la définition de procédures optimales de passation des marchés, dans des modélisations inspirées de la théorie des enchères (du Marais, 2003 ; Mougeot et Nagelen, 1993). Elle pose comme prémisse qu'un marché attribué selon une procédure optimale sera nécessairement exécuté en conséquence. Or, les conditions de l'exécution des marchés publics ne sont pas déterminées entièrement par la procédure de passation.

A cet égard, l'étude empirique des dépassements de coûts dans l'exécution des contrats administratifs, particulièrement des marchés publics, permet de montrer que les clauses contractuelles doivent être considérées à la lumière de la jurisprudence administrative qui s'y rapporte. Il donne également l'opportunité de comparaisons internationales qui conduisent à montrer l'existence d'idiosyncrasies institutionnelles.

Il a été mis en évidence, à l'issue d'une analyse statistique des arrêts du Conseil d'Etat rendus entre 1964 et 2001 en matière de contentieux de l'exécution du contrat administratif, que la position du juge administratif consiste à régler au cas par cas les affaires, en considération du standard de l'équilibre du contrat (Kirat, Marty, Vidal, 2003 ; Vidal, 2005). Le raisonnement des juges du Conseil d'Etat consiste à considérer conjointement le contenu des clauses du marché, les faits matériels invoqués par les parties, la jurisprudence antérieure, et le critère du bouleversement de l'« économie du contrat ». Nous avons mis en évidence l'absence de relations stables entre les faits matériels invoqués (par exemple : une grève retardant l'exécution, une nature du sol imprévue dans les marchés de travaux, une hausse soudaine des prix, la défaillance d'un sous-traitant, etc.) et les décisions du Conseil d'Etat. L'étude de ces décisions sur longue période a montré que les solutions données par la juridiction administrative suprême sont orientées davantage par le contenu des moyens du pourvoi (c'est-à-dire le contenu de la requête) que par toute autre variable. Néanmoins, le caractère restrictif de l'admission par le Conseil d'Etat des prétentions des entreprises à obtenir une compensation des surcoûts rencontrés dans l'exécution des marchés publics est clairement apparu. Ce fait empirique permet de tirer des enseignements pour l'analyse économique et la doctrine juridique.

Pour l'analyse économique, la jurisprudence du Conseil d'Etat ne permet pas d'établir l'existence d'une théorie, même implicite, de l'allocation des risques dans l'exécution du contrat administratif ; néanmoins, elle montre l'importance des clauses contractuelles, notamment des cahiers des prescriptions techniques, et des moyens du pourvoi dans l'interprétation des obligations des parties. Ce fait empirique est nettement en contradiction avec les approches théoriques affirmant le primat des arrangements négociés sur le recours aux tribunaux (Coase, 1960). Alors que ces dernières mettent 
l'accent sur les ajustements négociés à distance du formalisme du contrat, l'étude de la jurisprudence administrative montre l'enracinement profond de la régulation de l'exécution des marchés publics et des délégations de service public dans les documents constitutifs du contrat.

Pour la doctrine juridique, les faits empiriques mis en évidence aboutissent à fortement nuancer la portée pratique des théories de l'imprévision et des sujétions imprévues, évoquées précédemment. En effet, ces théories définissent logiquement les conditions dans lesquelles la survenance d'aléas imprévisibles ou de conditions qui rendent l'exécution du contrat plus onéreuse que prévue ex ante donnent droit à compensation financière, sous la forme soit d'une indemnité pour charge extracontractuelles, soit d'une révision du prix du marché. En fait, la faible fréquence des décisions d'admission des demandes dans ce sens constitue un trait structurel de la jurisprudence du Conseil d'Etat.

Il importe de signaler que le contentieux de l'exécution du contrat administratif ne constitue qu'une partie, certes importante, du contexte institutionnel de la régulation des surcoûts dans les marchés publics. Le paysage institutionnel de la régulation de ces conditions économiques de l'exécution est configuré par des textes réglementaires et des directives d'application émises par le Premier ministre ou le Ministre de l'économie, par les institutions dotées d'une capacité de contrôle des marchés publics. L'ensemble des règles et des institutions dessine un régime de réglementation au sein desquels les juges administratifs se situent, parmi d'autres intervenants. La considération de règles particulières et d'institutions prises indépendamment des autres auxquelles elles sont fonctionnellement liées porte un risque de compréhension partielle, voire biaisée, des mécanismes de régulation de l'exécution des marchés publics. Par conséquent, les comparaisons internationales de régimes de réglementation demande la mise en œuvre d'une perspective économico-juridique institutionnelle, qui situe l'observation de règles ou d'institutions particulières dans les interdépendances fonctionnelles qui les lient à d'autres.

La mise en œuvre d'une telle perspective dans la comparaison des dispositifs de régulation des dépassements de coûts dans les marchés de la Défense a permis de définir des «styles » réglementaires qui interdisent de comparer des règles locales entre elles. Nous pouvons citer, à titre d'exemple significatif, la règle commune aux Etats-Unis et à la France selon laquelle un marché d'armement postérieurement au développement (à fort contenu en risques) du matériel, doit être conclu sous une règle de prix forfaitaire (Fixed Price). Si cette doctrine de fixation des prix est commune aux pouvoirs publics français et américain, le contexte institutionnel dans lequel elle se situe est différente dans les deux cas. En effet, dans le cas des Etats-Unis, nous avons établi que la règle fonctionne en s'appuyant sur trois piliers qui lui sont fonctionnellement liés :

- une formalisation approfondie des recommandations économiques adressées aux fonctionnaires en charge des marchés, par le Code des marchés publics (Federal Acquisition Regulation) lui-même,

- une infrastructure de capacités d'audit, d'analyse économique et financière, utilisable par les organes qui passent des marchés,

- $\quad$ une juridiction administrative spécialisée dans le contentieux des marchés de la défense, qui se dote d'une jurisprudence claire et fortement orientée vers l'évaluation économique, financière et comptable des différends entre les autorités militaires et les fournisseurs d'équipements de défense.

Dans le cas français, l'environnement des marchés conclus à prix forfaitaire permet de faire en sorte qu'un contrat à prix forfaitaire fixé ex ante donne lieu à un règlement en dépassement de la limite fixée; l'absence structurelle de contentieux porté devant les juridictions administratives en matière de marchés de la défense, en dépit de désaccords fréquents entre les autorités publiques et les entreprises du secteur, témoigne de l'existence de mécanismes de régulation différents de ceux que l'on a pu 
constater aux Etats-Unis. La particularité de la régulation française réside dans l'existence d'un contrôle a priori des projets de marchés de la Défense, par la commission spécialisée des marchés compétente, et dans la pratique d'un contrôle de l'exécution par les seuls services techniques de l'Etat. La rationalité du contrôle de l'exécution par les services techniques étant orientée vers l'objectif de disponibilité des équipements, ils ont la capacité de susciter de la part des acteurs du financement et du règlement des marchés la mobilisation de ressources budgétaires supplémentaires afin de permettre l'achèvement des programmes d'armement ou l'exécution des prestations de maintenance des matériels par les entreprises (Kirat et Bayon, 2004).

\section{CONCLUSION}

En guise de conclusion, deux questions nous semblent devoir mériter attention.

En premier lieu, l'étude de la portée économique du droit peut être située au niveau des architectures institutionnelles des systèmes de réglementation. Alors que l'analyse économique du droit s'attache aux conséquences microéconomiques de règles de droit particulières, et que l'économie de la réglementation se focalise sur la justification économique de l'intervention réglementaire, nous avons retenu ici un point de vue inspiré de l'Institutional Law and Economics qui met l'accent sur les logiques d'action des institutions de réglementation et invite prendre en considération l'activité des tribunaux et des organes de régulation. Elle conduit également à s'appuyer sur des matériaux judiciaires (Korobkin, 2001) pour saisir la dynamique d'évolution de la réglementation publique et comprendre les modalités de sa mise en œuvre.

En second lieu, la mise en œuvre des règles ne se réduit pas à une simple opération d'application de dispositifs juridiques prescrivant des conduites ou des normes de manière univoque. Dans le secteur électrique, les réformes réglementaires menées depuis une dizaine d'années donnent lieu à des processus de construction de nouveaux dispositifs, dans lesquels les actions menées devant les autorités en charge de la concurrence sont d'une grande importance. Quant aux marchés publics, nous avons établi que l'observation de la mise en œuvre des règles du Code des marchés publics implique de prendre en considération les textes techniques, souvent de faible valeur normative du point de vue juridique, ainsi que la jurisprudence administrative.

\section{REFERENCES}

Allais, M. (1943), A la recherche d'une discipline économique, réédité comme Traité d'économie pure, Imprimerie Nationale, 1952.

Auby, J.-B., T. Kirat, F. Marty, L. Vidal (2003), Le contenu et la portée économiques des règles de droit. Application à la répartition des risques dans les contrats administratifs, rapport à la mission de recherche Droit et Justice, octobre, 336 p. (à paraître à la Documentation française, avec une préface de Marceau Long)

Baumol W., J. Panzar, R. Willig (1982), Contestable Markets and the Theory of Industry Structure, Harcourt, New York.

Bazzoli, L. (1999), L'économie politique de John R. Commons. Essai sur l'institutionnalisme en sciences sociales, L'Harmattan, coll. Etudes l'économie politique.

Bazzoli, L. et T. Kirat (2003), "À propos du réalisme en économie des institutions et ses implications sur l'analyse des fondements juridiques des transactions économiques: Commons versus Williamson", Economie appliquée, $\mathrm{n}^{\circ}$ 3, pp. 171-210.

Boiteux, M. (1949), "De la tarification des pointes de demande", Revue Générale de l'Electricité, tome 58, n 8, août, pp. $321-340$

Boiteux M., (1956), "Sur la gestion des monopoles publics astreints à l'équilibre budgétaire", Econometrica, January. 
Borenstein, S. (2002), "The Trouble with Electricity Markets: Understanding California Restructuring Disaster", Journal of Economic Perspectives, 16, pp. 191-211.

Bouckaert, B. and G. de Geest (dir.) (2000), Encyclopedia of Law and Economics, Edward Elgar.

Boy, L. (s.d.), (2000), Les pouvoirs de régulation de l'ART, rapport à la mission de recherche Droit et Justice, juillet.

Coase, R. (1960), "The Problem of Social Cost", Journal of Law and Economics, n 3, pp. 1-44.

Cohen, E. (1995), "L'Europe entre le marché et la puissance. Le cas des politiques de l’industrie. Spécialisation ; technologie, concurrence, commerce extérieur", in Y. Mény, P. Muller et J.-L. Quermonne, Politiques Publiques en Europe, L'Harmattan, Paris, 352p., pp. 183-202.

Commons, J.R. (1910), "How Wisconsin Regulates her Public Utilities", reprinted in M. Rutherford, W.J. Samuels (Eds), John.R. Commons:Selected Essays, Routledge, 1996.

Commons, J.R. (1924), Legal Foundations of Capitalism, New York, Macmillan.

Covalevski, M. A. (1995), "The use of accounting information in governmental regulation and public administration: The impact of John R. Commons and early institutional economists", the Accounting Historians Journal, June.

Cramton, E. (2003), "Electricity Market Design: The Good, the Bad and the Ugly", Proceedings of the Hawaii International Conference on System Sciences, January.

Custos, D. (2002), "Agences indépendantes de régulation américaines (IRC) et autorités administratives indépendantes françaises (AAI). L'exemple de la Federal Communications Commission (FCC) et de l'autorité de régulation des télécommunications (ART)", Politiques et Management Public, vol 20, nº 1.

Deakin, S. and S. Pratten (1999), "Reinventing the Market? Competition and Regulatory Change in Broadcasting", Journal of Law and Society, vol. 26, n³, September, pp.323-350.

Du Marais, B. (2003), Droit Public de la Régulation Economique, Dalloz, Paris, 640p.

Fitoussi, J.-P. (2003), EDF, le marché et l'Europe. L'avenir d'un service public, Fayard, Paris.

Glachant, J.-M. (2000), "Les pays d'Europe peuvent-ils reproduire la réforme électrique de l'Angleterre: Une analyse institutionnelle comparative", Economie et Prévision, vol 4, nº 145.

Jeammaud, A. (1993), "Les règles juridiques et l'action”, Recueil Dalloz, Chr.

Joskow, P.L. and J. Tirole (2004), "Reliability and Competition in Electricity Markets", IDEI Working Paper, Toulouse, May.

Kirat, T. et D. Bayon (2004), Contrats d'acquisition, maintenance et coût global de possession : comparaisons dans le domaine aéronautique entre la France, le Royaume-Uni, les Etats-Unis et l'OTAN, rapport de recherche à l'Observatoire économique de la Défense (ministère de la Défense), juin, 214 p. et annexes.

Kirat T., D. Bayon et H. Blanc (2003), Maîtriser les coûts des programmes d'armement : une analyse comparative de la réglementation des marchés industriels d'armement en France, au Royaume-Uni et aux Etats-Unis, Les rapports de l'Observatoire Economique de la Défense, La Documentation Française, Paris, 348 p

Kirat, T. et F. Marty (2003), "Les logiques économiques du contrôle a priori des marchés publics : les avis des commissions spécialisées des marchés", in J.-B. Auby, T. Kirat, F. Marty, L. Vidal, Le contenu et la portée économiques des règles de droit. Application à la répartition des risques dans les contrats administratifs, rapport à la mission de recherche Droit et Justice, octobre.

Kirat, T., F. Marty et L. Vidal (2003), "Analyse des arrêts du Conseil d'Etat sur les sujétions imprévues et l'imprévision dans les marchés publics et les délégations de service public", in J.-B. Auby, T. Kirat, F. Marty, 
L. Vidal, Le contenu et la portée économiques des règles de droit. Application à la répartition des risques dans les contrats administratifs, rapport à la mission de recherche Droit et Justice, octobre.

Korobkin, R. (2001), "Empirical Scholarship in Contract Law: Possibilities and Pitfall", Research Paper, $n^{\circ} 01-22$, UCLA School of Law.

Laffont, J.-J. (2000), "Etapes vers un Etat moderne : une analyse économique", rapport $\mathrm{n}^{\circ} 24$ du Conseil d'Analyse Economique, actes du colloque de décembre 1999, L'Etat et la gestion publique, La documentation française, juin

Laffont, J.-J. and J. Tirole (1993), A Theory of Incentives in Procurement and Regulation, MIT Press, Cambridge Ma.

McAfee, R.P. and McMillan, J. (1986), "Bidding for Contracts: A Principal-Agent Analysis", Rand Journal of Economics, volume 17, $\mathrm{n}^{\circ}$ 3, pp. 326-338.

Marimbert, J. (2001), Chronique dans Les Cahiers Juridiques de l'Electricité et du Gaz, décembre

Marty, F. (2002), Pluralité et dynamique des conventions de réglementation : une analyse économique de la jurisprudence du Conseil de la Concurrence relative au secteur de l'électricité, Thèse de doctorat es Sciences Economiques, Ecole Normale Supérieure de Cachan, décembre

Mercuro, N. (2000), "La common law, l'efficience et la Law and Economics institutionnaliste aux Etats-Unis", in Kirat et Serverin (dir.), Le droit dans l'action économique, CNRS Editions.

Mercuro, N., S. Medema et W.J. Samuels (2000), "Institutional Law and Economics", in Bouckaert, B. and G. de Geest (dir.), Encyclopedia of Law and Economics, Edward Elgar.

Mougeot, M. et N. Naegelen (1993), Les marchés publics : règles, stratégies, politiques, Economica, Paris, 230p.

Peltzman, S. (1989), "The Economic Theory of Regulation after a Decade of Deregulation", Brookings Paper Econ. Act. Microeconomics.

Perrot, A. (1995), "Ouverture à la concurrence dans les réseaux", Economie et Prérision, nº 119.

Polinski, M. and S. Shavell (2000), "The Economic Theory of Public Enforcement of Law", Journal of Economic Literature, vol. XXXVIII, March, pp. 45-76.

Posner, R.. (1992), Economic Analysis of Law, Little Brown, 4th edition, Boston.

Scherer, M. (1964), The Weapons Acquisition Process: Economic Incentives, Harvard Business School Press.

Shavell, S. (1984), "Liability for Harm vs. Regulation of Safety", Journal of Legal Studies, vol. 13, pp. 357-374.

Shavell, S. (1993), "The Optimal Structure of Law Enforcement", Journal of Law and Economics, vol. XXXVI, April, pp. 255-287.

Stigler, G.J., (1992), "Law or Economics?", Journal of Law and Economics, vol. XXXV, October, pp.455-468.

Vidal L. (2005), L'équilibre financier du contrat dans la jurisprudence administrative, Bruxelles, éditions Bruylant.

Weber M. (1922), Economie et Société. L'organisation et les puissances de la société dans leur rapport avec l'économie, trad. fra. éditions Pocket, 1995 